\title{
Activation of number facts in bilinguals
}

\author{
CHERYL FRENCK-MESTRE \\ University of Provence, Aix-en-Provence, France \\ and \\ JYOTSNA VAID \\ Texas A\&M University, College Station, Texas
}

\begin{abstract}
Two experiments examined the effect of the presentation format of numbers-digits versus word format in the first and in the second languages of bilinguals-on mental arithmetic. Speed of number-fact retrieval and the presence of interference produced by numbers that were either numerically close to or associatively related to the correct answers of stored arithmetic problems (e.g., $2+5$ and $7 \times 8$ ) were compared across formats. The verification of true problems was increasingly slower and less accurate from the digit condition to the second-language condition. Interference was produced by both types of incorrect answers in the digit and first-language conditions, whereas in the second-language condition, it was constrained to answers that were numerically close to correct answers. Together, the results suggest that the retrieval of arithmetic facts and the automatic spreading of activation within the network of numerical facts are not only language-sensitive, but format-sensitive in general.
\end{abstract}

The present research examined bilingual subjects' performance on tasks designed to provide insight into the storage and retrieval of arithmetic knowledge. To date, relatively little psycholinguistic research has been conducted on bilinguals' mathematical abilities in their two languages. What little that has been done has examined bilinguals' intentional manipulation of numbers and has generally found a language bias of some sort.

Specifically, two studies of the speed of mental arithmetic in bilinguals have shown a first-language advantage when bilinguals were confronted with their two languages simultaneously (Marsh \& Maki, 1976; McClain \& Huang, 1982). This result confirms two bilingual survey reports (Kolers, 1968; Vaid, Darkwah, \& Menon, 1992) that revealed a strong preference for performing mental arithmetic in the first language, the language in which number facts are generally learned. Nonetheless, this first-language advantage is not systematically observed. In one study (McClain \& Huang, 1982), it was found that when languages were blocked, the difference between mental calculation speed in the first and in the second languages was not reliable. In that study, it was suggested that the first-language advantage found under mixed-language conditions is due to switching between encoding and/or response systems. Because switching is not required in blocked-language conditions, the language effect should not be apparent, for actual calculation should not take a different amount of time in one or the other language once the numbers have been encoded in that lan-

Correspondence should be addressed to C. Frenck-Mestre, Centre de Recherche en Psychologie Cognitive, Université de Provence, 29 avenue Robert Schuman, 13621 Aix-en-Provence, Cedex, France (e-mail: crepco@frmop11). guage. Note, however, that yet another study (Tamamaki \& Sridhar, 1990) revealed a first-language advantage for mental arithmetic even under blocked-language conditions. The strength of the advantage was affected by the bilinguals' proficiency in their second language, with more proficient bilinguals showing a lesser difference in performance between their two languages.

Other research has revealed that bilinguals' language preference for mental calculation is determined by the word length of number words in their two languages (Elliott, 1991; Ellis, 1992; Ellis \& Hennelly, 1980). That is, if the digit names of one language take less time to articulate than those of another language, the former will be the preferred language for mental calculation. This effect of articulatory rate is accounted for in the terms of Baddeley's (1986) model of working memory, which posits that the capacity of working (short-term) memory is temporally limited. Whichever language has shorter number names will be more conducive to mental calculation, because more numbers can be held in memory and, for a given problem, the load placed on working memory during actual calculation will be lighter (see Ellis, 1992). ${ }^{1}$ In support of this argument, it has been widely documented that digit span (i.e., the number of single digits that can be simultaneously retained in short-term memory), is related to the articulatory rate of digit names in a given language (cf. Ellis, 1992, for a review).

Although bilinguals may consciously prefer to mentally calculate in one or the other of their two languages, the question remains as to whether numerical knowledge (i.e., stored number facts) is limited to that one language. In the above-mentioned studies, bilinguals were required to actively perform mental arithmetic. That is, they were required to mentally solve multiple-digit problems (e.g., 
$X+Y+Z=$ ?) in each of their two languages. Hence, factors such as differences in articulatory rate between the two languages, or practice with the second language, especially with numbers in that language, may in part explain the difference in mental calculation speed (Ellis, 1992; Magiste, 1982; Tamamaki \& Sridhar, 1990). If one examines the retrieval of numerical knowledge-that is, stored facts such as multiplication tables and simple addition-it is not immediately apparent that language primacy would play an important role. Indeed, arithmetic facts should, in principle, be retrieved from long-term memory. As such, one could argue that language should not affect the speed of number-fact retrieval for two reasons. First, number facts may be internally represented in an "abstract, format-free" manner (Sokol, GoodmanSchulman \& McCloskey, 1989; but see Campbell \& Clark, 1988; Clark \& Campbell, 1991; Gonzalez \& Kolers, 1982). Second, the retrieval of number facts should not involve any calculation and hence should not place any load on short-term (working) memory, in which the effects of articulatory rate appear. In support of the latter argument, one study (Ellis, 1992), with bilingual children, showed that performance was sensitive to language when the task involved mentally adding several digits but not when the task was to perform simple multiplication. It is important to note, however, that the children performed the tasks in only one of their two languages and that the comparisons between languages were between subjects. It is not certain that within-subject comparisons would reveal the same pattern of results, especially with adult bilinguals who have had years of practice with number facts such as multiplication problems in their preferred language.

The present study examined bilinguals' performance on numerical tasks that have been shown, in monolinguals, to tap into stored numerical knowledge. The tasks employed were designed to test for the presence of interference produced by numbers that were either numerically close to or associatively related to the correct answers of problems taken from the multiplication table and from simple addition problems. This type of interference is generally taken as evidence of an automatic spread of activation from the number activated by a simple arithmetic problem to other numbers within the network of stored numerical facts. That the activation of simple arithmetic facts is at least partially automatic (i.e., irrepressible; Posner \& Snyder, 1975) has been demonstrated in recent monolingual studies (LeFevre, Bisanz, \& Mrkonjic, 1988; Zbrodoff \& Logan, 1986). For example, subjects cannot avoid mentally adding two visually presented numbers although it is not required by task demands (LeFevre et al., 1988). Furthermore, when asked to verify the sum of a problem, subjects take longer to reject a false value that is the product of the problem as opposed to a false value that is unrelated to the problem (Winkleman \& Schmidt, 1974), although subjects can attenuate this associative confusion effect depending on task demands (Zbrodoff \& Logan, 1986). Concerning the spread of activation, it has been clearly demonstrated that when a given number is activated in memory-for example, the sum/product of an arithmetic problem-activation spreads from this number to neighboring numbers, with the result that it is harder to reject false answers that are numerically close to the correct sum/product than to reject false answers that are distant from the correct sum/product (Ashcraft \& Battaglia, 1978; Ashcraft \& Stazyk, 1981; Stazyk, Ashcraft, \& Hamann, 1982).

We extended these paradigms to a bilingual situation to determine whether or not the above-mentioned effects would occur in a like manner for numbers presented as spelled-out words in the first and second languages as for numbers presented in digit format. In Experiment 1, the effects of interference produced by false solutions to arithmetic problems were examined by means of an arithmetic verification task (in which the subjects were required to mentally verify the answers to simple arithmetic problems). In Experiment 2, a Stroop-type version of the arithmetic verification task was used to test for associative confusion effects produced by the activation of number facts. Both experiments also provided a measure of verification latencies to true problems. Thus, the experiments allowed us to examine the effect of the format of presentation of numbers on the activation of numerical facts, on the spread of activation, and on the speed of number-fact retrieval.

\section{EXPERIMENT 1}

In Experiment 1, an arithmetic verification task was used. Bilingual subjects were required to mentally add the two numbers of a visually presented pair of single digits (e.g., $3+4)$, and to decide whether a subsequently presented probe was the sum of the pair. The probe either was the sum of the pair or was numerically different from the true sum by \pm 1 or 2 (small split) or by \pm 5 or 6 (large split). The experiment enabled two types of comparisons. First, the time needed to mentally solve simple addition problems was compared across three presentation formats-digit format and each of the bilinguals' two languages-by examining the verification latencies to true problems. In line with previous bilingual research, we predicted that verification times would be slower for problems presented in the second language than in the first language (Marsh \& Maki, 1976). On the basis of previous monolingual and bilingual results (Frenck-Mestre \& Vaid, 1991; LeFevre et. al., 1988), we also expected the digit condition to produce the fastest verification latencies of all three presentation formats. Second, the effect of interference from numbers that were numerically close to correct responses was compared for all three presentation formats (digits, and the first and second languages) by examining the pattern of response times across all problem types. For digits, we expected response times to be faster overall for true-sum probes than for nonsum probes. Moreover, we expected slower rejection latencies for nonsum probes that were close in value to the true sum (small split) than for nonsum probes whose value was farther 
away from the true sum (large split). These predictions are derived from previous monolingual studies in which a network model of mental arithmetic is promoted, showing that the time needed to reject false problems decreases as the numerical difference between the false and the correct sum increases (Ashcraft \& Battaglia, 1978; Ashcraft \& Stazyk, 1981; Stazyk et al., 1982), due to a spread of activation from correct sums to neighboring numbers.

By comparing the digit and first-language conditions, we could determine whether or not similar patterns of interference would be observed for numbers presented as words and as digits. A priori, we could expect similar effects for these two formats, given the results of a monolingual study on arithmetic fact retrieval (LeFevre et al., 1988). In that study, the predicted effects were observed in a like manner whether the stimulus material was presented as digits or as number words. The extent to which interference occurred in the second language was examined by comparing the patterns of performance in the first and second languages.

Lastly, the activation of number facts was examined at two stimulus onset asynchronies (SOAs) of 120 and $240 \mathrm{msec}$ between problem onset and probe onset. This manipulation allowed us to determine whether the effect of split would be more apparent at the shorter of the two SOAs. In fact, previous work has suggested that automatic effects due to the activation of number facts are extremely transient in nature (LeFevre et al., 1988).

\section{Method}

Subjects. Fifteen American exchange students, studying at a French university, voluntarily participated in the experiment. Mean length of residency in France at the time of participation was 6 months. To participate in the exchange program, subjects were required to have completed at least 2 years of college French in the United States (mean was 2 years at college level and 3.5 years at secondary level) and to be capable of following college courses conducted in French. Individual questioning of the subjects by the experimenter revealed that all considered themselves to be relative novices in French. However, it should be noted that all were "functionally fluent" in that they could carry on a normal conversation in French and conduct both academic and private activities (paying bills, etc.) in that language. All subjects were late bilinguals, having learned French after early childhood. The subjects ranged in age from 19 to 22 years and included 6 men and 9 women.

Stimuli. A problem consisted of an initial pair of distinct numbers separated by a plus sign (e.g., $2+5$ ), followed by a probe number (e.g., 7). The subjects' task was to decide whether the probe was the sum of the initial number pair. The numbers, both in the initial pairs and as probes, ranged from 1 to 9 excluding 6 (which is written "six" both in English and in French). ${ }^{2}$ Three experimental conditions and a control condition were employed. In the experimental conditions, the probe was either the sum of the pair (sum) or a number that differed from the sum by \pm 1 or 2 (split $\pm 1,2$ ) or by \pm 5 or 6 (split $\pm 5,6$ ). ${ }^{3}$ All other types of numerical relations between the number pair and probe were avoided. A positive response was required for the true-sum probes, whereas a negative response was required for the split $\pm 1,2$ and split $\pm 5,6$ probes. Twelve number pairs were used across the three experimental conditions. For example, the initial number pair $2+7$ was followed by the probes 9 (sum), 8 (split $\pm 1,2$ ), and 3 (split $\pm 5,6$ ). Another twelve number pairs were used exclusively as control trials. In the control trials, the probe was the sum of the initial pair; thus a positive response was required. The control trials were used to equate the number of negative and positive trials, and were not included in the analyses.

The subjects solved 16 different problems (four control trials, with 4 problems for each experimental condition: sum, split $\pm 1,2$, and split $\pm 5,6$ ) in each of three format blocks (digits, number words in the first and second languages). All experimental problems were seen in all formats and paired with each of the three probes; however, a given subject saw a problem in only one condition according to a Latin square design. All problems were seen at two different SOAs between the number pair and the probe (120 and $240 \mathrm{msec}$ ). The subjects saw the same format block of problems twice, once at each SOA. In total, the subjects participated in six blocks of 16 trials.

Apparatus and Procedure. Display of stimuli and response recording were controlled by a microcomputer. Problems were displayed in green against a dark background in $5 \times 5 \mathrm{~mm}$ lowercase characters.

The experiment was divided into two main blocks, one for each SOA. The order of SOA blocks and of format blocks within an SOA block was counterbalanced across subjects. The order of problems within each format block was independently randomized for each subject. Each block began with four practice problems that were not among the problems in the block.

Each trial began with the presentation of a central fixation point, followed by a number pair. The number pair was presented horizontally, and the two numbers were separated by a plus sign. The number pair was displayed for 120 or $240 \mathrm{msec}$, depending on the SOA condition, and was replaced immediately by the probe, which remained centrally displayed until the subject had responded. The intertrial interval was 2 sec.

The subjects were tested individually. They were told that they would see two numbers, followed by a probe number. The subjects were informed of the format of presentation of numbers before each block. The subjects were instructed to indicate manually, using designated "yes" and "no"' keys on the keyboard, whether or not the probe was the sum of the initial number pair. No feedback was given.

\section{Results}

Response times. Response time (RT) data for correct trials were first subjected to a 3 (probe type: sum, split $\pm 1,2$, split $\pm 5,6) \times 3$ (format: digit, first language number words, second language number words) $\times 2$ (SOA: 120 and $240 \mathrm{msec}$ ) repeated measures analysis of variance (ANOVA). This analysis was intended to reveal the pattern of probe interference for each of the three presentation formats. The results are summarized in Table 1.

The global analysis of response times revealed main effects of all three factors. The main effect of SOA condition $[F(1,14)=54.16, p<.001]$ revealed slower $\mathrm{RT}$ in the $120-\mathrm{msec}$ than in the $240-\mathrm{msec}$ SOA condition $(1,239$ and $1,003 \mathrm{msec}$, respectively). The effect of format $[F(2,28)=57.93, p<.001]$ revealed increasing $\mathrm{RT}$ from the digit condition $(762 \mathrm{msec})$ to the first-language condition $(1,209 \mathrm{msec})$ to the second-language condition $(1,393 \mathrm{msec})$. This effect will be examined more closely below, taking only true-sum probes into account. Concerning probe type, mean RTs for true-sum probes, for small-split $( \pm 1,2)$ probes, and for large-split $( \pm 5,6)$ probes were $1,029,1,276$, and $1,058 \mathrm{msec}$, respectively $[F(2,28)=29.73, p<.001]$ 
Table 1

Mean Response Times (RT; in Milliseconds) and Corresponding Error Rates Obtained in Experiment 1 as a Function of Probe Type, Format of Presentation of Number Stimuli, and Stimulus Onset Asynchrony (SOA)

\begin{tabular}{|c|c|c|c|c|c|c|}
\hline \multirow[b]{3}{*}{ Probe } & \multicolumn{6}{|c|}{ Format of Presentation } \\
\hline & \multicolumn{2}{|c|}{ Digit Format } & \multicolumn{2}{|c|}{ 1st Language } & \multicolumn{2}{|c|}{ 2nd Language } \\
\hline & RT & $\%$ Error & RT & $\%$ Error & RT & \% Error \\
\hline \multicolumn{7}{|c|}{ SOA $120 \mathrm{msec}$} \\
\hline Sum & 733 & .05 & 1,161 & .07 & 1,513 & .23 \\
\hline Split $\pm 1,2$ & 930 & .10 & 1,605 & .15 & 1,654 & .30 \\
\hline Split $\pm 5,6$ & 845 & .02 & 1,281 & .02 & 1,432 & .10 \\
\hline \multicolumn{7}{|c|}{ SOA $240 \mathrm{msec}$} \\
\hline Sum & 635 & .03 & 985 & .05 & 1,149 & .15 \\
\hline Split $\pm 1,2$ & 780 & .05 & 1,219 & .08 & 1,466 & .17 \\
\hline Split $\pm 5,6$ & 650 & .02 & 1,001 & .02 & 1,142 & .03 \\
\hline
\end{tabular}

There was a trend for the interaction between probe type and format $[F(4,56)=2.22, p<.08]$. Independent analyses were performed on the data for each of the three formats to examine the effect of probe interference within each format. In the digit condition, the main effect of probe was significant $[F(2,28)=11.07, p<.001]$. Orthogonal contrasts of means (Winer, 1971) revealed that mean RTs were faster to sum probes (positive trials) than to the two nonsum probe conditions [negative trials; $F(1,28)=8.56, p<.01]$ and that small-split probes produced slower RTs than did large-split probes $[F(1,28)=$ $13.58, p<.001]$. The same effects were observed in the first-language condition: main effect of probe type $[F(2,28)=22.59, p<.001]$, sum versus nonsum probes $[F(1,28)=25.77, p<.001]$, and small-split versus large-split probes $[F(1,28)=19.40, p<.001]$. In the second-language condition, a somewhat different pattern of results was observed. The main effect of probe was significant $[F(2,28)=6.43, p<.005]$; however, mean RTs were not faster overall to sum than to nonsum probes $[F(1,28)=1.69$, n.s. $]$. This reflects the fact that sum and large-split probes produced similar RTs, which were substantially faster than RTs to small-split probes. Small-split probes produced slower RTs than did large-split probes $[F(1,28)=11.17, p<.01]$. None of the above patterns of results were significantly modified by SOA.

A partial analysis was performed on the RT data obtained for correct, positive trials (true-sum probes) to test for the effect of format of presentation on the speed of mental arithmetic. Mean RTs were 684, 1,073, and $1,331 \mathrm{msec}$ for problems presented in digit format, in the first language, and in the second language, respectively $[F(2,28)=36.21, p<.001]$. Orthogonal contrasts of means confirmed the predictions that verification latencies of simple addition problems would be faster for problems presented in digit than in word format $[F(1,28)=$ $61.03, p<.001]$ and for problems presented in the first than in the second language $[F(1,28)=11.39, p<.01]$. This pattern of results was true at both SOAs. There was, nonetheless, a main effect of SOA $[F(1,14)=30.16, p<$
.001 ], due to slower mean RTs in the 120- than in the 240-msec SOA condition, and an interaction effect between SOA and format $[F(2,28)=3.36, p<.05]$. A test of the partial interaction $[F(1,28)=6.17, p<.02]$ showed that the difference between RTs in the two SOA conditions was substantially larger for problems presented in the second language ( $364 \mathrm{msec}$ ) than for problems presented in the first language $(176 \mathrm{msec})$ and in digit format $(98 \mathrm{msec})$.

Errors. Inspection of error data gave no indication of speed-accuracy tradeoffs. The analysis of data obtained for positive (true-sum) trials revealed only a main effect of format $[F(2,28)=9.90, p<.001]$; error rates were $4 \%, 6 \%$, and $19 \%$ for digits, the first language, and the second language, respectively. The analysis of data obtained for negative (split $\pm 1,2$ and split $\pm 5,6$ ) trials revealed main effects of all three factors. A higher percentage of errors was found for small-split than for large-split probes $[14 \%$ and $3 \%$, respectively; $F(1,14)=13.70$, $p<.002]$, at the $120-$ than at the $240-\mathrm{msec}$ SOA [11\% and $6 \%$, respectively; $F(1,14)=4.38, p<.05$ ], and in the second-language condition as compared with the firstlanguage and digit conditions $[15 \%, 6 \%$ and $5 \%$, respectively; $F(2,28)=9.42, p<.001]$.

\section{Discussion}

Experiment 1 sought to answer two questions. The first question was whether bilinguals would show sensitivity to the format of presentation when asked to mentally verify the solution to simple addition problems. The answer is straightforward. Bilinguals were slower to respond to problems presented in their second language than in their first language. In addition, verification latencies were globally slower for problems presented in word format than in digit format. The difference between mean verification latencies was actually greater between digits and the first language than between the first and the second languages (although error rate was substantially higher in the second language than in the first language and for digits). Thus, it may well be that practice with numbers in a given format is partially responsible for differences in processing time (see Vaid \& Frenck-Mestre, 1991). Just as monolinguals are more accustomed to processing numbers in digit format than in word format, bilinguals have had, in general, greater exposure to numbers in their first language than in their second language.

The second issue at stake was whether the interference from incorrect answers that has been observed for digits in addition (Ashcraft \& Stazyk, 1981) and in multiplication (Stazyk et al., 1982) would be apparent when numbers were presented as number words and, more specifically, as number words in the first and in the second languages. The interference produced by digits has generally been taken as an indication of the spread of activation, from correct sums to neighboring numbers, on presentation of an arithmetic problem. It is noteworthy that the amount of interference produced generally decreases 
as the numerical distance between the true and incorrect answer increases (Ashcraft \& Battaglia, 1978; Ashcraft \& Stazyk, 1981; Stazyk et al., 1982).

Our results provide evidence of interference from smallsplit probes, as opposed to large-split probes, for all three format conditions. That is, we observed interference for digits and found it also in both the first and second languages of the subjects. If one considers only the RTs to false responses, averaged across the two SOAs, the effect of interference is practically indistinguishable across the two language conditions ( 271 and $273 \mathrm{msec}$ in the first and second languages, respectively). These results thus confirm previous results showing similar patterns of automatic activation of numbers presented as digits and as number words in the first language (see LeFevre et al., 1988), and extend the finding to the second language of bilinguals.

We can note, nonetheless, that the overall pattern of results, taking RTs to true answers into account, reveals a pattern for the second-language condition that was somewhat different from that in the first-language and digit conditions. In both of the latter conditions, we obtained the habitual effect of split, wherein response times were overall faster for true-sum probes than for nonsum probes, and small-split probes produced interference when compared with large-split probes. In the second language, truesum probes did not produce faster overall response times than did nonsum probes, because there was no reliable difference in response time between large-split probes and correct sums (in fact, mean RT was slightly faster to largesplit probes than to correct sums). The overall pattern of results obtained in the second language differs not only from the digit and first-language conditions in our own experiment, but also from previous monolingual studies with similar levels of split (Ashcraft \& Stazyk, 1981). Caution thus seems warranted before concluding that performance in the second language was in all respects the same as that in the first language and for digits.

The aim of Experiment 2 was again to test for automatic effects of number processing, for numbers presented as digits and as words in the first and second languages.

\section{EXPERIMENT 2}

Experiment 2 examined the activation of number facts in an arithmetic version of the Stroop paradigm. As in Experiment 1, bilingual subjects verified the solution of simple arithmetic problems presented in digit format and in each of their two languages. In this experiment, false solutions were either unrelated to the problem (e.g., $2+5=9$ ) or were associatively related to the problemthat is, they would have been true had the subject performed multiplication instead of addition, and vice versa (e.g., $2+5=10 ; 2 \times 5=7$ ). As in Experiment 1, there were two different comparisons of particular interest. First, by comparing the verification latencies for true solutions presented in the first and in the second languages, we could determine if resolution time was affected by the language of presentation. Second, within each of the two languages, the comparison of latencies for associatively related solutions with those for unrelated solutions allowed us to determine whether the automatic activation of associated number facts (i.e., the automatic spread of activation) occurred in either or both of the bilinguals' languages. The digit condition was employed as a baseline condition in which the above-mentioned associative confusion effects were expected to occur on the basis of previous results with monolinguals (Winkleman \& Schmidt, 1974; Zbrodoff \& Logan, 1986).

\section{Method}

Subjects. Fifteen English-French bilinguals, taken from the same population that was used in Experiment 1, voluntarily participated. None had taken part in the earlier experiment.

Stimuli. Problems were presented horizontally in the form of $a \times b$ and $a+b$ in the multiplication and addition tasks, respectively. Problems were followed by the presentation of a response, $c$. Three different types of trials were defined by the nature of $c$. In true trials, $c$ was the true answer to the problem. In associatively related trials, $c$ was the sum of the problem in the multiplication task (e.g., $7 \times 5=$ 12 ) and the product of the problem in the addition task (e.g., $7+5=$ 35 ). In neutral trials, $c$ was mathematically unrelated to the problem. The value of $c$ for the neutral trial of a problem was the value of $c$ for the associatively related trial $\pm 1-3$, such that the absolute difference from the true answer (split) was equated for these two conditions.

Experimental trials. The operands were the single digits from 1 to 9 , excluding the digit 6 (which is written "six" in English and in French) ${ }^{4}$ Zero was excluded as an operand given that zero problems can be solved by reference to a rule (e.g., $0+N=N$; $0 \times N=0$ ), and zero problems have been shown to induce erratic performance (Sokol, McCloskey, Cohen, \& Aliminosa, 1991; Stazyk et al., 1982). Tie problems (e.g., $3 \times 3$ ) were excluded because they have been shown to behave differently from nontie problems (Groen \& Parkman, 1972). Within these constraints, there are a total of 28 different pairs of problems sharing the same operands (e.g., $2+8$ and $8+2,7+5$ and $5+7$, etc.), whereas no two pairs have the same product. From these pairs, 21 were chosen such that no more than two different pairs shared the same sum (e.g., $9+2$ and $8+3$ ). This latter constraint was imposed given that the previous retrieval of an answer (product) has been shown to produce interference on subsequent trials (Campbell, 1990). The split between true answers and negative trials varied across problems, given that sums replaced products and vice versa for associative lures. However, the size of split was equated across the two arithmetic tasks (e.g., $7+3=21$ and $7 \times 3=10$ both have a split of \pm 11 ; $8+9=72$ and $8 \times 9=17$ both have a split of \pm 55 , etc.).

The 21 pairs of problems were split into two symmetrical halves. One half was tested in the addition task, and the other half was tested in the multiplication task (e.g., $2+8,7+5$, and $8 \times 2,5 \times 7$, etc.), with the set of 21 problems counterbalanced across tasks. In each task, each problem was presented three times: once in each of three blocked formats (digit, English number names, and French number names) and associated with a different type of trial (true, associatively related, and neutral). For example, a given subject saw " $5+4$ " followed by " 9" (digit, true), "five + four" followed by "twenty" (English, associatively related), and "quatre + cinq" followed by "dix-neuf" (French, neutral). For a given problem, type of trial was counterbalanced across formats in the two tasks. There were 7 trials of each type for a total of 21 different experimental trials per blocked format. Problems were distributed across trial type in such a way that problems sharing the same true sum were presented with different answers within a format block (e.g., $2+9=$ 
11 and $8+3=24$ ). All problems were thus seen in all conditions: however, a subject saw a given problem in only one condition.

Control trials. An additional 21 true trials, 7 per format, were presented to equate the number of positive and negative trials in each format block ( 14 in each case). The same problems were used in the multiplication and addition tasks. The operands were the single digits 0 to 9 . These problems were selected in accordance with the restriction that, within a format block, no more than two problems share the same true sum (experimental and control problems confounded) and no true response (sum or product) was actually repeated. These problems were not included in the analyses.

To summarize, each subject saw six blocks of 28 different problems ( 21 experimental [ 7 true, 7 lures, and 7 neutral] and 7 control [true]). Three blocks (one digit, one English, and one French) were presented in each of the two arithmetic tasks (addition vs. multiplication).

Apparatus and Procedure. These were identical to those outlined in Experiment 1, with the following exceptions. First, the experiment was divided into two main blocks in accordance with the arithmetic task (addition vs. multiplication). Second, the two operands in a problem were separated by either a plus sign or an uppercase $X$ with adjacent blank characters, depending on the arithmetic task. Third, the problem was displayed for $300 \mathrm{msec}$ before being replaced immediately by the probe. Lastly, the subjects were told that they would see an arithmetic problem, followed by an answer. and were instructed to verify the answer as quickly as possible. Before each block, the subjects were informed of the type of arithmetic task and of the format that numbers would be presented in.

\section{Results}

Response times for correct trials and error data were subjected to independent ANOVAs. For both RT and error data, separate analyses were performed on data for positive trials (true probes) and for negative trials (associatively related and neutral probes). The results are summarized in Table 2.

The 3 (format: digit, first language, second language) $\times 2$ (task: addition, multiplication) analysis performed on the RT data obtained for positive trials (true probes) revealed a main effect of format $[F(2,28)=113.76, p<$ $.001]$. Mean RTs were 688,945 , and $1,459 \mathrm{msec}$ in the digit and in the first- and second-language conditions, re-

Table 2

Mean Response Times (RT; in Milliseconds) and Corresponding Error Rates Obtained in Experiment 2 as a Function of Probe Type, Format of Presentation of Number Stimuli, and Arithmetic Task

Format of Presentation

\begin{tabular}{|c|c|c|c|c|c|c|}
\hline \multirow[b]{3}{*}{ Probe } & \multicolumn{6}{|c|}{ Format of Presentation } \\
\hline & \multicolumn{2}{|c|}{ Digit Format } & \multicolumn{2}{|c|}{ 1st Language } & \multicolumn{2}{|c|}{ 2nd Language } \\
\hline & RT & $\mathrm{PE}$ & RT & PE & RT & PE \\
\hline \multicolumn{7}{|c|}{ Addition } \\
\hline Correct answers & 703 & .03 & 907 & .05 & 1,399 & .16 \\
\hline Associative lures & 778 & .04 & 1,016 & .06 & 1,318 & .10 \\
\hline Neutral lures & 712 & .04 & 939 & .03 & 1,381 & .15 \\
\hline \multicolumn{7}{|c|}{ Multiplication } \\
\hline Correct answers & 673 & .04 & 985 & .05 & 1,518 & .21 \\
\hline Associative lures & 734 & .30 & 1,043 & .04 & 1,317 & .04 \\
\hline Neutral lures & 759 & .02 & 1,010 & .03 & 1,342 & .11 \\
\hline
\end{tabular}

Note-PE $=$ percent error. spectively. Orthogonal contrasts of means confirmed that RT was faster in the digit than in the word-format conditions $[F(1,28)=130.29, p<.001]$, and in the first than in the second language $[F(1,28)=97.24, p<.001]$. This pattern was not significantly different in the two arithmetic tasks, nor was there a main effect of task $[F(1,14)=$ 2.15 , n.s.]. A similar pattern of results was found in the analysis of error data, which revealed an effect only of format $[F(2,28)=8.79, p<.001]$, with $3 \%, 5 \%$, and $19 \%$ error in the digit and in the first- and second-language conditions, respectively.

The 2 (probe: associatively related, neutral) $\times 3$ (format) $\times 2$ (task) analysis performed on RT data for negative trials did not reveal an effect of probe type $(F<1)$ or of task $(F<1)$. There was a main effect of format $[F(2,28)=72.78, p<.001]$, which was modified by an interaction with probe $[F(2,28)=5.05, p<.01]$. The analysis of error data also revealed an interaction effect of probe and format $[F(2,28)=18.81, p<.001]$. Independent analyses were performed on the RT and error data for each of the three formats to examine the effect of probe interference within each format.

In the digit condition, the analysis of RT did not reveal an effect of probe $(F<1)$. However, probe and arithmetic task tended to interact $[F(1,14)=4.08, p<.06]$. Tests of simple effects revealed that associatively related probes, in contrast to neutral probes, produced interference in the addition task [778 vs. $712 \mathrm{msec}$, respectively; $F(1,14)=5.28, p<.04]$, but not in the multiplication task (734 vs. $759 \mathrm{msec}$ for associatively related and neutral probes, $F<1$ ). The analysis of error rates also revealed an interaction between probe and task $[F(1,14)=$ $113.21, p<.001]$. Here, interference from associatively related probes was observed in the multiplication task (30\% and $2 \%$ error for associative and neutral probes, respectively) but not at all in the addition task (4\% error for both types of probes). In sum, interference from associatively related probes was observed for RTs in the addition task and on error rate in the multiplication task.

In the first-language condition, the analysis of RT revealed a main effect of probe $[F(1,14)=6.50, p<.02]$, reflecting the interference produced by associatively related as opposed to neutral probes. No other effects approached significance, nor were there any effects on error rate, which was low overall $(4 \%)$.

In the second-language condition, the analysis of RT revealed no significant effects: probe $[F(1,14)=2.58$, n.s.]; probe $\times$ task $(F<1)$. The analysis of error rates showed only an effect of probe $[F(1,14)=4.69, p<.05]$, which was due to a higher error rate in the neutral $(13 \%)$ than in the associatively related $(7 \%)$ probe condition.

\section{Discussion}

The results of Experiment 2 confirm the finding that bilinguals are slower and less accurate in their second language than in their first language when they are required 
to actively retrieve arithmetic facts. Poorer performance was observed in the second language in a like manner for both addition and multiplication. It can be noted, however, that, as in Experiment 1, verification latencies were in general slower for problems presented in word format than in digit format. The difference between the firstlanguage condition and the digit condition, if not as great as that between the two languages, was still large. Thus, practice with a given format has a strong impact on performance. Interestingly, however, for true problems, there was no notable difference in error rates between the firstlanguage condition and the digit condition, whereas a substantial drop in performance was observed in the secondlanguage condition. We can speculate that although our bilingual subjects took longer to verify solutions presented in their first language than as digits, undoubtedly because of habit, they did in fact "know" their number facts in that language, which was apparently not the case for the second language.

The results of Experiment 2 also lead us to conclude that associative confusion effects, resulting from the automatic activation of number facts, appear to be constrained to the first language, the language in which our bilingual subjects learned arithmetic. No evidence of interference from false answers that were associatively related to the problem (e.g., $2+5=10$ ) was observed in the second language, although this type of interference was observed for both digits and the first language. In the second language, the only reliable effect was on error rate, and the data actually revealed higher error rate for neutral than for associatively related trials. The reason for this effect is not immediately apparent; however, it would indeed not appear to be due to the automatic activation of a response that was arithmetically related to the operands in the problem.

In the first language, interference was observed in both arithmetic tasks and affected only response times (error rate was low). In the digit condition, interference was observed in both arithmetic tasks; however, rather interestingly, it affected only error rate in the multiplication task and only RT in the addition task. The asymmetrical effect on RTs in the digit condition is in line with previous monolingual results (Zbrodoff \& Logan, 1986) showing greater interference from the irrelevant process in addition than in multiplication. In that study, no analysis of error rate was given. In our study, we have evidence of associative confusion in a like manner for addition and multiplication in the digit condition, if one takes both dependent variables (RT and error rate) into account.

\section{GENERAL DISCUSSION}

The present set of experiments investigated bilingual subjects' mathematical abilities in their two languages. Specifically, two types of comparisons were made. On the one hand, we compared performance in the two languages for the intentional retrieval of arithmetic facts. On the other hand, we examined the patterns of interference produced, in each of the bilinguals' languages, by incorrect answers that were numerically close to and/or associatively related to the correct answers of simple arithmetic problems as a means of testing for automatic spreading activation within the network of number facts.

Concerning the retrieval of stored numerical knowledge, the results from Experiments 1 and 2 clearly demonstrate that bilinguals are at a disadvantage when they are required to perform in their second language instead of in their first (the language in which number facts were learned). This was true even though languages were blocked. Although one bilingual study has shown that language differences disappear under blocked conditions (McClain \& Huang, 1982; but see Ellis, 1992, and Tamamaki \& Sridhar, 1990), it should be noted that both the time constraints and the task demands of our study differed from those of any previous study with bilingual subjects. First, the problems were presented at a much more rapid rate than in previous studies with bilinguals. Second, the task involved verifying the solutions to simple addition problems (with sums under 20) and problems taken from the multiplication table (up to $9 \times 8$ ), rather than mentally adding several digits. The task was thus more one of retrieving number facts from memory than of mentally solving multidigit arithmetic problems. Our results show that these facts are not only languagesensitive but sensitive to format in general. In both experiments, there was a large difference in response times between the first language and the digit format as well as between the first and the second languages. Results of a similar nature, showing that the external format of numbers affects processing time on numerical tasks, have been taken as evidence for the hypothesis that numerical symbols are stored in multiple formats that are integrated in memory (Campbell \& Clark, 1988; Clark \& Campbell, 1991; Gonzalez \& Kolers, 1982), as opposed to the hypothesis that arithmetic fact retrieval is mediated by internal representations that are independent of the external format in which they are presented (Sokol et al., 1991).

Although our results clearly show language-dependent effects of arithmetic fact retrieval, we cannot make as strong a claim about the automatic spread of activation. In Experiment 1, the results revealed interference from incorrect sums that were numerically close to the correct answers of arithmetic problems, thereby suggesting the existence of an automatic spreading of activation within the mental network of numbers comparable to that described for words (Anderson, 1983; Collins \& Loftus, 1975). This interference was observed not only for numbers presented as digits but for numbers presented in the first and second languages of subjects as well. ${ }^{5}$ In Experiment 2, we also observed interference effects, whereby subjects took longer to reject false values that were the products of addition problems or the sums of multiplication problems than to reject false values that were unrelated to problems. However, although these associative confusion effects were observed for numbers presented in digit format and as words in the first language, no such 
interference was apparent for numbers presented as words in the second language.

The apparent discrepancy between the results of Experiments 1 and 2 may possibly be explained by differences in the language sensitivity of relationships within the network of numerical facts. Interference was observed in the second language for numbers that were numerically very close to the correct response (i.e., " 7 " for the problem $3+5$; Experiment 1), but not for associatively related incorrect responses (i.e., " 12 "' for the problem $3+4$; Experiment 2). It is conceivable that there would be less of the latter type of interference in our subjects' second language because all of the subjects had learned their arithmetic facts in their first language. Multiplication and simple addition would have been more highly overlearned in the first than in the second language, since late bilinguals (those who learned their second language after puberty) do not relearn arithmetic facts in their second language. Therefore, although these bilinguals may be lured by incorrect answers that differ from correct answers by only a small amount, as seen in Experiment 1, they may not be as easily led into associative traps in their second language, because these associations may be weaker or perhaps take longer to activate than was allowed by the 300-msec SOA that we employed. Preliminary results of our own, from an experiment using the same materials and addition task described in Experiment 2, but with highly proficient Spanish-English bilinguals, again suggest that associative interference operates for problems presented in digit format and in the bilinguals' first-learned language (Spanish, in this case), but not in the secondlearned language (Vaid, Frenck-Mestre, \& Balfour, 1992). Although further investigation is warranted, the latter results strengthen the hypothesis that the discrepancy between the results of Experiments 1 and 2 may be due to the types of relationships employed, with associative confusion effects perhaps being restricted to the first language of bilinguals.

In both of our experiments, the subjects' verification of arithmetic facts was considerably worse in their second language than in their first. We should like to argue that the lower performance observed in the second language is truly tied to arithmetic, rather than to the manipulation of number words in general. Support for this argument can be found in three different bilingual studies in which various tasks were used. In one study (FrenckMestre \& Vaid, 1991), a number-matching task was employed in which subjects' task was to decide if a probe number (e.g., 7) had been presented in an immediately preceding number pair (e.g., 23 ). Thus, the task involved mentally retaining numbers (presented as digits and in each of the bilinguals' languages) but did not involve intentional mental arithmetic. It is important to note that both the stimulus materials and presentation rate that were used were quite comparable to those of the present study. The results of the earlier study revealed no reliable difference in accuracy between the first and second languages
(3.5\% and $5.7 \%$ overall error, respectively), and a difference in processing time between the two languages of less than $100 \mathrm{msec}$ (798 vs. $869 \mathrm{msec}$ for the first and second languages, respectively). The differences in performance due to language were thus much smaller than those observed in the present two experiments (both numerically and proportionately with regard to RT). In another study of bilinguals, no reliable difference between languages was found when the task was to make a lexical decision to number words presented in the two languages (Frenck-Mestre \& Vaid, 1992). Lastly, results from a bilingual study on incidental recall revealed that language of presentation was not a particularly salient feature for number-word stimuli (Vaid \& Frenck-Mestre, 1991). The results from all three of the above studies thus argue for the relative nonsalience of language in the processing of numbers when the task is not one of mental arithmetic.

Regarding our claim that language does have an impact on mental arithmetic, two potential worries about our results should be dispelled. First, the comparison of the subjects' performances in their two languages does not lead us to believe that the subjects were actively translating from their second language to their first to complete the tasks. We can note that in both Experiments 1 and 2 , the subjects were less accurate in their second language than in their first. This was especially the case for trials in which the true answer was presented. Had subjects been translating, one would have expected the slower response times that were observed for the second language, but not the higher error rates. Of course, it is quite impossible to ensure that subjects never translate when performing a task in their weaker language, and our results cannot entirely rule out this possibility. If the subjects did translate either the problem or the answer before responding, and were sometimes inaccurate, this could explain the high error rate. We can note that the subjects did not report that they engaged in translation; they claimed to be performing the task, albeit rather unsuccessfully, in their second language. The high error rates in the second language for true trials again suggests that the subjects were particularly hard put when required to verify arithmetic facts in that language. It would appear that once the subjects had encoded the problem in their second language, they were then at a loss as to what the answer should be. This again argues against the automaticity of retrieval of arithmetic facts in the second language. Second, the difference in the patterns of results obtained in our bilinguals' first and second languages strengthens the argument that the language effects we obtained reflect actual retrieval differences in addition to differences in encoding time. Were the effects we obtained simply due to slower encoding in the second language, we should not have obtained the radically different pattern of results that was observed in the second language when compared with the first language. Moreover, as mentioned above, the differences in performance that we observed between the first and second languages were substantially larger in the 
present study than those found in a very similar previous study (Frenck-Mestre \& Vaid, 1991), which did involve encoding numbers but did not involve performing arithmetic.

It is important to note that the overall slower processing time in the second language may conceivably have masked any automatic effects, which are short-lived by definition. Also, the high error rates observed in the second language attest to the fact that our subjects were experiencing difficulty with the tasks in that language, and this difficulty undoubtedly had some impact on the encoding stage as well as on the retrieval stage of processing. It is important to determine whether the pattern of effects obtained in the present study would obtain with more experienced bilinguals, who may show lesser differences in processing time between their two languages.

To conclude, the present set of experiments provides an interesting account of late bilinguals' mental arithmetic capacities in their two languages. First, we have clear evidence that language plays a nonnegligible role in the retrieval of stored arithmetic knowledge, with bilinguals being at a disadvantage in their second language. This finding complements those of previous studies showing that multidigit mental computation is sensitive to the format in which numbers are presented. Second, our results show similar patterns of interference from incorrect answers presented in digit format and in the first language of bilinguals, whereas interference was present but more constrained in the second language. Taken together, our results suggest that both the retrieval of arithmetic facts and the automatic spreading of activation within the network of numerical facts are at least language-sensitive, if not languagedependent. Further bilingual research, with comparisons between other languages and with more balanced bilinguals, is warranted given the implications of these findings for both monolingual and bilingual research.

\section{REFERENCES}

ANDERSON, J. R. (1983). A spreading activation theory of memory. Joumal of Verbal Learning \& Verbal Behavior, 22, 261-295.

AshCraft, M. H., BattaGLia, J. (1978). Cognitive arithmetic: Evidence for retrieval and decision processes in mental addition. Journal of Experimental Psychology: Human Learning \& Memory, 4, 527-538.

Ashcraft, M. H., \& Stazyk, E. H. (1981). Mental addition: A test of three verification models. Memory \& Cognition, 9, 185-196.

BadDeley, A. (1986). Working memory. Oxford: Oxford University Press.

Campbell, J. I. D. (1990). Retrieval inhibition and interference in cognitive arithmetic. Canadian Joumal of Psychology, 44, 445-464.

Campbell, J. I. D., Clark, J. (1988). An encoding-complex view of cognitive number processing: Comment on McCloskey, Sokol and Goodman (1986). Journal of Experimental Psychology: General, 117, 204-214.

Clark, J., C Campbell, J. I. D. (1991). Integrated versus modular theories of number skills and acalculia. Brain \& Cognition, 17, 204-239.

Collins, A. M., \& LofTus, E. F. (1975). A spreading activation theory of semantic processing. Psychological Review, 82, 407-428.

ElLIOTT, J. M. (1991). Is language important in mental arithmetic? Singapore Journal of Education, 11, 35-44.
Eltus, N. C. (1992). Linguistic relativity revisited: The bilingual wordlength effect in working memory during counting, remembering numbers and mental calculation. In R. J Harris (Ed.), Cognitive processing in bilinguals (pp. 137-155). Amsterdam: North-Holland/Elsevier.

Ellis, N. C. \& HenNelly, R. A. (1980). A bilingual word-length effect: Implications for intelligence testing and the relative ease of calculation in Welsh and English. British Journal of Psychology, 71, 43-51.

Frenck-Mestre, C., \& VAid, J. (1991, November). Mental manipulation of numbers by bilinguals. Poster presented at the 32nd Annual Meeting of the Psychonomic Society, San Francisco.

FrencK-MEstre, C., \& VAID, J. (1992). Language as a factor in the identification of ordinary words versus number words. In R. J. Harris (Ed.), Cognitive processing in bilinguals (pp. 265-281). Amsterdam: North-Holland/Elsevier.

GonZAlez, E., \& Kolers, P. A. (1982). Mental manipulation of arithmetic symbols. Journal of Experimental Psychology: Learning, Memory, \& Cognition, 8, 308-319.

Groen, G. J., Parkman, J. M. (1972). A chronometric analysis of simple addition. Psychological Review, 79, 329-343.

Hoosaln, R. (1984). Experiments on digit spans in the Chinese and English languages. In S. R. Kao \& R. Hoosain (Eds.), Psychological studies of the Chinese language. Hong Kong: Chinese Language Society of Hong Kong.

Kolers, P. (1968). Bilingualism and information processing. Scientific American, 218 (3), 78-86.

LeFevre, J.-A., Bisanz, J., Mrkonjic, L. (1988). Cognitive arithmetic: Evidence for obligatory activation of arithmetic facts. Memory \& Cognition, 16, 45-53.

MAGiste, E. (1982). The importance of language strategy in simple arithmetic. Educational Psychology, 2, 159-166.

MARSH, L. G., \& MAKI, R. H. (1976). Efficiency of arithmetic operations in bilinguals as a function of language. Memory \& Cognition, 4, 459-464.

MCClain, L., \& HuANG, J. Y. S. (1982). Speed of simple arithmetic in bilinguals. Memory \& Cognition, 10, 591-596.

PoSNer, M. I., \& SNYder, C. R. (1975). Attention and cognitive control. In R. L. Solso (Ed.), Information processing and cognition: The Loyola Symposium. Hillsdale, NJ: Erlbaum.

Sokol, S. M., Goodman-Schulman, R. McCloskey, M. (1989). In defense of a modular architecture for the number-processing system: Reply to Campbell and Clark. Journal of Experimental Psychology: General, 118, 105-110.

Sokol, S. M., McCloskey, M., Cohen, N. J., \& Aliminosa, D. (1991). Cognitive representations and processes in arithmetic: Inferences from the performance of brain-damaged subjects. Journal of Experimental Psychology: Learning, Memory, \& Cognition, 17, 355-376

Stazyk, E. H., Ashcraft, M. H., Hamann, M. S. (1982). A network approach to mental multiplication. Joumal of Experimental $P_{S y-}$ chology: Learning, Memory, \& Cognition, 8, 320-335.

TAMAMAKI, K., \& SRIDHAR, S. N. (1990). Relative efficiency of bilinguals' arithmetic operations in their two languages as a function of language use. Unpublished manuscript, State University of New York at Stony Brook.

VAID, J., DARKwah, A., \& Menon, R. (1992). Correlates of language preference for mental arithmetic in Spanish-English bilinguals (Working Paper Series, Report II, Number II). College Station, TX: Texas A\&M University, Race and Ethnic Studies Institute.

VAID, J., \& FrenCK-MESTRE, C. (1991). Incidental memory for format of presentation of number stimuli: Evidence from monolinguals and bilinguals. Brain \& Cognition, 17, 272-284.

Vaid, J., Frenck-Mestre, C., \& Balfour, S. (1992, November). Does language figure in numerical fact retrieval? Poster presented at the 33rd Annual Meeting of the Psychonomic Society, St. Louis.

Winer, B. J. (1971). Statistical principles in experimental design. (2nd ed.). New York: McGraw-Hill.

Winkleman, H. J., \& SCHMidt, J. (1974). Associative confusions in mental arithmetic. Joumal of Experimental Psychology, 102, 734-736. 
Zbrodoff, N. J., \& Logan, G. D. (1986). On the autonomy of mental processes: A case study of arithmetic. Joumal of Experimental PSychology: General, 115, 118-130.

\section{NOTES}

1. Note that this line of reasoning has led some authors to claim that languages that have rapid articulatory rates, such as Chinese, are in general more suited to mental arithmetic than are languages with slower articulatory rates (see Hoosain, 1984, cited in Elliott, 1991; Ellis, 1992).

2. All initial number pairs had sums that were 9 or below.

3. The average absolute difference between the probe and each of the two numbers in the pair (e.g., for the pair 34 with probe 7 , the mean absolute difference is 3.5 ) was controlled for across experimental conditions $(3.4$ in the sum and split $\pm 1,2$ conditions and 3.5 in the split $\pm 5,6$ condition), given that this factor has been found to affect response times in a task similar to the present one (LeFevre et al., 1988).

4. It should be noted that the mean word length of the number-names used (both operands and problem answers) was roughly equivalent in English and in French, which thus avoids a possible confounding of effects due to rate of articulation (see Ellis, 1992).

5 . We can note, however, that although the patterns of results observed for digits and for first-language number words fit well with existing data (Ashcraft \& Battaglia, 1978; Ashcraft \& Stazyk, 1981; Stazyk et al., 1982), the pattern observed for second-language number words is only partially coherent with previously obtained results.

(Manuscript received June 8, 1992; revision accepted for publication May 14, 1993.) 